\title{
Die Zukunft der Psychiatrie und des Psychiaters*
}

\author{
The Future of Psychiatry and Psychiatrists
}

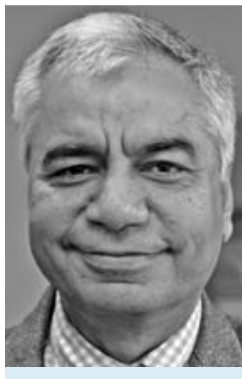

Dinesh Bhugra

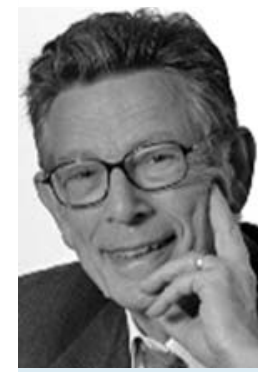

Norman Sartorius
Wir wurden eingeladen, entgegengesetzte Positionen in einer Debatte zu der These einzunehmen, dass der Psychiater als Beruf und die Psychiatrie als medizinische Disziplin vom Verschwinden bedroht ist. Wir fanden jedoch bald heraus, dass wir in allen wesentlichen Punkten in dieser Angelegenheit übereinstimmen. Dies ließ es schwierig erscheinen, gegensätzliche Positionen zu formulieren. Deshalb verwandelte sich die Debatte in eine Aufzählung der Dinge, die zu tun sind, wenn der Psychiater als Beruf und die Psychiatrie als Fach überleben sollen. Während wir beide hinsichtlich der Bedeutung der nachfolgend aufgelisteten Punkte übereinstimmen, mag es andere geben, die unsere Empfehlungen für weder wichtig noch notwendig halten. Dies könnte in der Tat eine Debatte generieren, an deren Ende klar wird, dass es Aufgaben gibt, die das Überleben der Psychiatrie als Disziplin und der Professionellen, die sie praktizieren, sichern.

Im vergangenen Vierteljahrhundert haben diverse Entwicklungen die Psychiatrie erschüttert:

- In Westeuropa und in den USA gab es einige größere medizinische und psychiatrische Skandale, die unter Psychiatern zu Demoralisierung und Entfremdung führten;

- es gab Berichte über aufregende Forschungsergebnisse hinsichtlich der Entschlüsselung der Hirnregionen und ihrer Funktionen, der Entwicklung der

* Übersetzung: Tilman Steinert, Ravensburg
Psychopharmakagenomik und der Neurowissenschaften der Emotionen;

- Forscher und Betroffenenorganisationen stellten fest, dass das Stigma psychischer Erkrankungen nicht abgenommen hat und dass Diskriminierung aufgrund von Stigma und chronischer Unterfinanzierung psychiatrischer Einrichtungen rund um den Globus große Herausforderungen für die Psychiatrie bleiben;

- der ökonomische Abschwung und seine Folgen fügten eine Reihe weiterer Probleme hinzu, ebenso wie die Politik mancher Regierungen. Die Bürokratie hat sich vermehrt und verbraucht mehr und mehr Mittel, welche der Krankenpflege zugeordnet waren;

- sich verändernde Patientenerwartungen und Probleme, Nachwuchs für die Psychiatrie zu gewinnen, sind in vielen Teilen der Welt zusätzlich Gegenstand der Sorge;

- verschiedene Berufsgruppen haben Verschreibungsrechte bekommen;

- zu den von außen kommenden Bedrohungen der Psychiatrie gehören auch die strikte Trennung der Arbeitsaufgaben zwischen unterschiedlichen Berufsgruppen und eine vermehrte Konsumhaltung aufseiten der Nutzer;

- auch interne Bedrohungen bestehen in erheblichem Maße, zum Beispiel die Selbststigmatisierung von Psychiatern und die zunehmende Super-Spezialisierung, die bedeutet, dass die Spezialisten für ihr Fachgebiet, aber nicht für die Profession als Ganzes sprechen und dass diejenigen, die Psychiatrie in der Breite betreiben, oft als zweitklassig angesehen und auch so behandelt werden.

Was kann also getan werden?

\section{Der Weg in die Zukunft}

Es kann kein Zweifel bestehen, dass dies in der Tat aufregende Zeiten für die Psychiatrie sind und dass die Psychiatrie eine glänzende Zukunft hat unter der Voraus- setzung, dass der Berufsstand in der Lage ist, eine Führungsrolle zu übernehmen. Wir schlagen vor:

1. Die Profession muss die fachlichen Qualifikationen im Hinblick auf technische Kompetenz, klare Kommunikation, Interessensvertretung für Patienten, uneigennützigen Rat an die Politik und die vernünftige Verwendung der verfügbaren Ressourcen erkennen und wertschätzen.

2. Wo Psychiater Rollen übernehmen, die kongruent mit den oben genannten professionellen Werten sind, sollten sie eine klare Führungsrolle übernehmen und ihre Fähigkeiten in Teamarbeit dazu nutzen, Einrichtungen zu entwickeln und zu gestalten, die angemessen, wirksam und kosteneffektiv sind.

3. Psychiater müssen einen Weg finden, mit einer Stimme zu sprechen. Gegenwärtig besteht über wesentliche Dinge keine Übereinstimmung, z. B. über die Dauer, die Methoden und die Inhalte der Weiterbildung (bis zum Facharzt und danach) und die optimalen Bedingungen der Behandlung und Versorgung. Die Profession muss mit einer einzigen klaren Stimme sprechen. Psychiater müssen auch die Grenzen ihrer Kompetenz und ihrer Verantwortung definieren. Gegenwärtig sind sie mit einer Vielzahl von Problemen beschäftigt, die von einer medikalisierten Form der Behandlung von Problemen des täglichen Lebens bis zur Gestaltung der sozialen Versorgung für schwerbehinderte Menschen, die gegenwärtig keine psychiatrische Erkrankung haben, reichen. Die Profession muss die Führung darin übernehmen, klar zu definieren, wofür wir stehen, was unsere Stärken sind und was wir tun sollten.

4. Psychiater sollten ihre Positionen und ihre Rollen definieren, die sie in öffentlichen Gesundheitsprogrammen spielen wollen. Längere Zeit haben sich Psychiater gescheut, Aufgaben zu Fragen der Prävention und der öffentlichen Gesundheitsfürsorge wahrzuneh- 
men, obwohl sie über Evidenz verfügen, dass viele psychische Störungen verhindert werden können und dass Frühinterventionen wirksam sind.

5. Psychiater sollten alle evidenzbasierten Behandlungen in ihr therapeutisches Arsenal integrieren und der Tendenz widerstehen, sich auf Psychopharmakotherapie zu beschränken. Leitlinien für die Therapie sind hilfreich und Psychiater müssen Pharmakotherapie mit Psychotherapie und sozialen Interventionen kombinieren, wo dies indiziert und angemessen ist.

6. Die Kompetenzen von Psychiatern sollten auch weiterhin diejenigen von Ärzten sein, die in der Lage und willens sind, sich mit körperlichen Krankheiten bei ihren Patienten zu beschäftigen. Die Integration von körperlicher und psychischer Gesundheit ist von überragender Bedeutung. In ihrer Eigenschaft als Ärzte müssen Psychiater die Fähigkeiten und Kompetenzen haben, körperliche Gesundheitsstörungen zu erkennen und angemessene Hilfe zu realisieren.

7. Psychiater müssen gegen die Medikalisierung von Problemen des täglichen Lebens eintreten, während sie umgekehrt sicherstellen müssen, dass tat- sächliche psychische Störungen behandelt werden. Psychiater sollten eine Meisterschaft in den Behandlungstechniken ihrer Disziplin erwerben und ihrer Banalisierung und der Tendenz, sie als simple Techniken darzustellen, entgegentreten. Psychiater sollten evidenzbasierte Behandlungen nutzen und sich an translationaler Forschung und in jedem Stadium klinischer Studien beteiligen.

8. Psychiatrische Einrichtungen der Zukunft sollten Patienten, den für ihre Versorgung zuständigen Professionellen und ihren Familien helfen, die Fähigkeiten des Selbstmanagements zu erlernen und dabei auch die Erfahrungen und Fähigkeiten anderer Patienten nutzen, die die Rolle als „Peer Support“-Mitarbeiter übernommen haben.

Psychische Erkrankungen werden weiter ein großes gesellschaftliches Problem darstellen und seine Lösung wird die Beiträge von Psychiatern erfordern, die kompetent in ihren Rollen der Diagnostik und Behandlung von psychischen Krankheiten sind und die bereit sind, eine Führungsaufgabe beim Umgang der Gesellschaft mit psychischen Erkrankungen übernehmen.
Sie haben eine eigene Meinung zu diesem Thema? Dann schreiben Sie uns an: psychiat-praxis@thieme.de!

\section{Korrespondenzadressen}

\section{Prof. Dr. Dinesh Bhugra}

President of the World Psychiatric Association (WPA), Emeritus Professor of Mental Health \& Cultural Diversity, Health Service \& Population Research Department, Institute of Psychiatry, Psychology \& Neuroscience, King's College London London SE5 8AF

dinesh.bhugra@kcl.ac.uk

\section{Prof. Dr. med. Dr. phil. Dr. h. c. mult.}

\section{Norman Sartorius}

Past President of the World Psychiatric Association (WPA), President Association for the Improvement of Mental Health Programmes (AMH)

14 chemin Colladon

1209 Geneva

Switzerland

sartorius@normansartorius.com

Bibliografie

Dol http://dx.doi.org/

10.1055/s-0035-1552738

Psychiat Prax 2015; 42: 413-414

(c) Georg Thieme Verlag KG

Stuttgart · New York

ISSN 0303-4259 